\title{
Determining the mass loss limit for close-in exoplanets: what can we learn from transit observations?
}

\author{
H. Lammer ${ }^{1}$, P. Odert ${ }^{2}$, M. Leitzinger ${ }^{2}$, M. L. Khodachenko ${ }^{1}$, M. Panchenko' ${ }^{1}$, Yu. N. Kulikov ${ }^{3}$, T. L. Zhang ${ }^{1}$, \\ H. I. M. Lichtenegger ${ }^{1}$, N. V. Erkaev ${ }^{4}$, G. Wuchterl ${ }^{5}$, G. Micela ${ }^{6}$, T. Penz ${ }^{6,7}$, H. K. Biernat ${ }^{1}$, J. Weingrill ${ }^{1}$, M. Steller ${ }^{1}$, \\ H. Ottacher ${ }^{1}$, J. Hasiba ${ }^{1}$, and A. Hanslmeier ${ }^{2}$ \\ 1 Space Research Institute, Austrian Academy of Sciences, Schmiedlstrasse 6, 8042 Graz, Austria \\ e-mail: helmut.lammer@oeaw.ac .at \\ 2 Institute for Physics, IGAM, University of Graz, Universitätsplatz 3, 8010 Graz, Austria \\ 3 Polar Geophysical Institute, Russian Academy of Sciences, Khalturina 15, 183010 Murmansk, Russian Federation \\ 4 Institute for Computational Modelling, Russian Academy of Sciences, and Siberian Federal University, Krasnoyarsk, \\ Russian Federation \\ 5 Thüringer Landessternwarte Tautenburg, Sternwarte 507778 Tautenburg, Germany \\ ${ }^{6}$ INAF - Osservatorio Astronomico, Piazza del Parlamento 1, 90134 Palermo, Italy \\ 7 On leave from the INAF - Osservatorio Astronomico, Palermo, Italy
}

Received 23 February 2009 / Accepted 31 July 2009

\section{ABSTRACT}

\begin{abstract}
Aims. We study the possible atmospheric mass loss from 57 known transiting exoplanets around F, G, K, and M-type stars over evolutionary timescales. For stellar wind induced mass loss studies, we estimate the position of the pressure balance boundary between Coronal Mass Ejection (CME) and stellar wind ram pressures and the planetary ionosphere pressure for non- or weakly magnetized gas giants at close orbits.

Methods. The thermal mass loss of atomic hydrogen is calculated by a mass loss equation where we consider a realistic heating efficiency, a radius-scaling law and a mass loss enhancement factor due to stellar tidal forces. The model takes into account the temporal evolution of the stellar EUV flux by applying power laws for F, G, K, and M-type stars. The planetary ionopause obstacle, which is an important factor for ion pick-up escape from non- or weakly magnetized gas giants is estimated by applying empirical power-laws.

Results. By assuming a realistic heating efficiency of about $10-25 \%$ we found that WASP-12b may have lost about $6-12 \%$ of its mass during its lifetime. A few transiting low density gas giants at similar orbital location, like WASP-13b, WASP-15b, CoRoT-1b or CoRoT-5b may have lost up to $1-4 \%$ of their initial mass. All other transiting exoplanets in our sample experience negligible thermal loss $(\leq 1 \%)$ during their lifetime. We found that the ionospheric pressure can balance the impinging dense stellar wind and average CME plasma flows at distances which are above the visual radius of "Hot Jupiters", resulting in mass losses $<2 \%$ over evolutionary timescales. The ram pressure of fast CMEs cannot be balanced by the ionospheric plasma pressure for orbital distances between 0.02-0.1 AU. Therefore, collisions of fast CMEs with hot gas giants should result in large atmospheric losses which may influence the mass evolution of gas giants with masses $<M_{\text {Jup }}$. Depending on the stellar luminosity spectral type, planetary density, heating efficiency, orbital distance, and the related Roche lobe effect, we expect that at distances between 0.015-0.02 AU, Jupiter-class and sub-Jupiter-class exoplanets can lose several percent of their initial mass. At orbital distances $\leq 0.015$ AU, low density hot gas giants in orbits around solar type stars may even evaporate down to their coresize, while low density Neptune-class objects can lose their hydrogen envelopes at orbital distances $\leq 0.02 \mathrm{AU}$.
\end{abstract}

Key words. planetary systems - planetary systems: formation

\section{Introduction}

The upper atmospheres of short periodic exoplanets are affected strongly by the X-ray and EUV radiation as well as by the dense plasma environment of their host stars. Therefore, these exoplanets experience high thermal (Lammer et al. 2003; Vidal-Madjar et al. 2003; Lecavelier des Etangs et al. 2004; Yelle 2004, 2006; Baraffe et al. 2004; Tian et al. 2005; Erkaev et al. 2007; Hubbard et al. 2007a,b; Lecavelier des Etangs 2007; Muñoz 2007; Koskinen et al. 2007; Penz et al. 2008a,b; Penz \& Micela 2008; Davis \& Wheatley 2009; Murray-Clay et al. 2009) and non-thermal (Erkaev et al. 2005; Khodachenko et al. 2007) atmospheric mass loss over their lifetime.
Khodachenko et al. (2007) studied the expected minimum and maximum possible atmospheric $\mathrm{H}^{+}$pick-up erosion of the "Hot Jupiter" HD209458b due to stellar Coronal Mass Ejections (CMEs) and concluded that hydrogen-rich gas giants, which orbit solar-like stars at distances $\leqslant 0.05 \mathrm{AU}$, would have been strongly eroded if the upper atmospheres were not protected by a magnetosphere. This magnetosphere should be strong enough so that the stellar plasma flow can be deflected at planetary distances where the total atmospheric pressure $P_{\text {tot }}$ is $\leq 10^{-4} \mathrm{dyn} \mathrm{cm}^{-2}$. These authors found that the stellar wind induced $\mathrm{H}^{+}$pick-up loss could erode a "Hot Jupiter" to its coresize if the planetary obstacle forms at distances where $P_{\text {tot }}$ is $>10^{-4} \mathrm{dyn} \mathrm{cm}^{-2}$. This non-thermal mass loss process depends 
on the strengths of the intrinsic magnetic moments. Grießmeier et al. (2004) estimated for slow rotating, tidally locked "Hot Jupiters" that their magnetic moments should be in the range between $0.005-0.1 \mathcal{M}_{\text {Jup }}$. From this study one can expect that weakly magnetized short periodic gas giants, which experience a Venus-like stellar plasma-atmosphere interaction, should exist.

Comparative spacecraft observations of Earth, Venus and Mars indicate a large variation of ionospheric density and ionopause location between solar minimum and maximum conditions (e.g. Evans 1977; Zhang et al. 1990; Kliore \& Luhmann 1991). All planetary bodies in the Solar System with substantial atmospheres (e.g. Earth, Venus, Mars, and Titan) produce ionospheres which expand above the exobase. Therefore, one can also expect that the extreme XUV exposed and hydrodynamically expanding thermospheres of "Hot Jupiters" will also have a strong ionized component, which may raise the ionopause to distances where the ion pick-up process could be less effective, as suggested by Khodachenko et al. (2007).

The aim of this paper is to estimate the initial mass of 57 known transiting exoplanets ${ }^{1}$ for which the stellar and planetary parameters are well determined. Furthermore, we investigate at what orbital distances hydrogen-rich gas giants or "Hot Neptunes" can maintain their initial atmospheric hydrogen inventories, and at what distance they could experience huge atmospheric mass loss. Additional to the thermal mass loss study, we investigate if the ionospheric pressure of non- or weakly magnetized "Hot Jupiters" can be strong enough to balance the ram and magnetic pressure of the dense stellar wind and CME plasma flow at planetary distances which are beyond the critical erosion level with its total atmospheric pressure of $\approx 10^{-4} \mathrm{dyn} \mathrm{cm}^{-2}$. Finally, we compare the efficiency of thermal and non-thermal mass loss processes with exoplanetary observations and previous mass loss estimations.

\section{Thermal atmospheric mass loss}

\subsection{Heating efficiency in a hydrogen-rich thermosphere}

Recently, Penz et al. (2008a) studied for the first time the EUV driven thermal atmospheric mass loss from the low density gas giant HD209458b over its evolutionary time period by applying a full hydrodynamic approach to its hydrogen thermosphere. These authors found that the thermal mass loss rate can be well approximated by a modified energy-limited formula which includes a mass loss enhancement factor due to the Roche lobe effect and a heating efficiency for the stellar EUV radiation.

The heating efficiency $\eta$ is conventionally defined as ratio of the net local gas heating rate to the rate of stellar radiative energy absorption. In the earlier studies by Lammer et al. (2003), Baraffe et al. (2004) and also in some recent studies, like those by Lecavelier des Etangs (2007), Hubbard et al. (2007a,b) and Davis \& Wheatley (2009), their authors applied the energy-limited equation with the assumed heating efficiency of $\eta=100 \%$. But as indicated by more detailed studies, the heating efficiency factor, if appropriately evaluated, would be considerably less than $100 \%$, which can substantially reduce the mass loss that was overestimated in the earlier studies. However, calculation of a realistic value of $\eta$ in a planetary atmosphere is very complex, which results in a wide scatter of the adopted heating efficiencies. For example, Watson et al. (1981) applied a

\footnotetext{
${ }^{1}$ Extrasolar Planets Encyclopedia status February 2009; http:// www . exoplanet.eu/
}

Table 1. EUV related heating processes and heating efficiencies $\eta$ within the hydrogen-rich thermosphere of Jupiter (after Waite et al. 1983).

\begin{tabular}{cc}
\hline \hline Heating process & $\eta[\%]$ \\
\hline Neutral heating & 9.26 \\
Electron heating & 7.92 \\
Vibrational energy & 6.23 \\
$\sum$ & 23.41 \\
Chemical heating $\left(\mathrm{H}_{2}^{+}\right)$ & 29.74 \\
Total $\sum$ & 53.15 \\
\hline
\end{tabular}

height-integrated average value of $\eta$ in a hydrogen-rich thermosphere of the early Earth of about $30 \%$, while Kasting \& Pollack (1983) assumed it to be $15 \%$ in a water-rich early atmosphere of Venus. A far more detailed analysis by Waite et al. (1983) has shown, however, that the average heating efficiency $\eta$ of the solar EUV radiation in the hydrogen dominated thermosphere of Jupiter is $\sim 53 \%$ (see Table 1 for details). Chassefière (1996a) again studied the EUV energy deposition and hydrodynamic escape of atomic hydrogen from an assumed hot $\mathrm{H}_{2} \mathrm{O}$-rich early Venusian atmosphere and found, in agreement with Kasting \& Pollack (1983), that for atmospheric hydrogen number density between $10^{10}-3 \times 10^{10} \mathrm{~cm}^{-3}$, the value of $\eta$ is $\leq 30 \%$ and may be closer to about $15 \%$.

Yelle (2004), in his mass loss study from a "Hot Jupiter", also modelled its neutral and ion chemical composition and found that $\mathrm{H}_{2}$ molecules are the dominant species in the lower thermosphere $\left(\leq 1.07 r_{\mathrm{pl}}\right)$, which is consistent with the Jovian atmosphere composition (Waite et al. 1983). Yelle (2004) also analyzed the EUV heating rate and related heating efficiency and found that the average value of $\eta$ is about $50-60 \%$ in the lower thermosphere, where the main gas is molecular hydrogen. Above these altitudes $\left(\geq 1.07 r_{\mathrm{pl}}\right), \mathrm{H}_{2}$ is thermally dissociated into atomic hydrogen and thus a part of the thermal energy of the gas that could drive atmospheric escape is spent instead on dissociation. Also at these altitudes $\left(\geq 1.07 r_{\mathrm{pl}}\right)$ strong photo-ionization of atomic hydrogen starts to dominate and one can expect that most of the absorbed EUV energy should drive escape. However, ionmolecular exothermic reactions, which should produce chemical heating, will not in fact deposit their energy as heat due to lower frequency of collisions between the reacting species in this region. Taking this consideration into account, one can conclude that the total EUV related heating efficiency in the upper layers of the "Hot Jupiters" thermosphere cannot be more than $\sim 20-30 \%$.

In confirmation of this, the heating efficiency $\eta$ modelled by Yelle (2004) above the ionospheric peak drops to a value of about $10-15 \%$, also in agreement with the earlier estimates by Chassefière (1996b). At still larger distances $\eta$ drops to values which are even $\leq 10 \%$. This very low heating efficiency is obtained because much of the absorbed stellar energy goes into ionization of hydrogen atoms and then is either lost through escape of $\mathrm{H}^{+}$ions, or upon recombination of $\mathrm{H}^{+}$where the chemical energy is converted to radiant energy, i.e., a photon, that escapes from the optically thin upper atmosphere. As a consequence, most of the stellar EUV energy absorbed by hydrogen atoms above the ionospheric peak escapes to space and does not contribute to local heating. This process differs from recombination of $\mathrm{H}_{2}^{+}$and/or $\mathrm{H}_{3}^{+}$in the lower thermosphere, which returns chemical energy to the atmosphere through the kinetic energy of the reaction products.

Furthermore, in a recent work by Murray-Clay et al. (2009) who studied the EUV driven mass loss from "Hot Jupiters" during their host stars' pre-main sequence stage (T-Tauri phase), it 
is shown that the heating efficiency changes with time. These authors discovered that for high EUV fluxes $\left(\geq 10^{4} \mathrm{erg} \mathrm{cm}^{-2} \mathrm{~s}^{-1}\right)$, Lyman- $\alpha$ cooling of the thermosphere becomes important. In this cooling process, Lyman- $\alpha$ radiation is emitted by neutral hydrogen atoms that are collisionally excited by electrons (Murray-Clay et al. 2009). These authors found that other cooling processes like thermal conduction, collisional ionization, radiative recombination, and free-free emission become negligible in such extreme EUV conditions.

Besides the EUV heating, atmospheric heating by X-rays may also become essential (Cecchi-Pestellini et al. 2006). $\mathrm{X}$-rays, due to their smaller absorption cross-sections, must deposit their energy deeper in the atmosphere where the gas is mostly molecular, unlike the EUV radiation, which is absorbed at higher altitudes where the atmosphere mostly consists of atomic and ionized hydrogen. Although the heating efficiency for X-rays originates from processes different those related to EUV radiation, in a fully molecular gas, according to Maloney et al. (1996), it may reach values of about 30-40\% and thus substantially contribute to lifting the dense gas out of the gravitational potential well of a planet. However, in a more recent article on that topic by Cecchi-Pestellini et al. (2009), these authors computed the X-ray heating efficiencies dependent on the energy of the incoming photons as functions of the absorbing column density and found lower X-ray heating efficiencies of about $10-20 \%$.

From the above discussion, it follows that the columnaveraged heating efficiency for a hydrogen-rich gas giant's atmosphere is most likely $<60 \%$ and possibly is closer to $25 \%$, or even less during the active evolutionary stage of the planet's young host star, due to Lyman- $\alpha$ cooling (Murray-Clay et al. 2009). Because the actual heating efficiency of a hydrogen atmosphere exposed to an extreme stellar environment is still poorly constrained, more studies of X-ray related thermospheric heating are needed. In view of this, we apply for our mass loss simulations the following values of $\eta: \eta=10 \%, 25 \%$ which are constant over time, and for comparison with previous studies (e.g., Lecavelier des Etangs 2007; Hubbard et al. 2007a,b; Penz et al. 2008a,b; Davis \& Wheatley 2009) $\eta=60 \%$ and $100 \%$. Because Lyman- $\alpha$ cooling becomes important during active young star epochs, we study the mass loss from hydrogen-rich gas giants at very close orbital distances assuming also time-dependent values of $\eta$, which are $10 \%$ during the early time when high EUV radiation dominates and later grow to $25 \%$, and even $60 \%$ after the flux values are less than $10^{4} \mathrm{erg} \mathrm{cm}^{-2} \mathrm{~s}^{-1}$.

\subsection{Method}

The maximum possible thermal atmospheric loss rate $L_{\mathrm{th}}$ can occur when all the absorbed stellar EUV and X-ray energy is converted to heat locally. This means that infra-red cooling (e.g., cooling by $\mathrm{CO}_{2}, \mathrm{H}_{3}^{+}$, etc.) and heat transport by thermal conduction can be neglected and the kinetic energy of the escaping flow is negligibly small when compared to the potential energy of the lifted gas. These conditions were originally assumed by Sekiya et al. (1980a,b, 1981) and Watson et al. (1981) who developed the first hydrodynamic models of atmospheric escape from hydrogen-rich atmospheres of primordial, Earth-like planets. Watson et al. (1981) also derived analytical formulae for the treatment of the flow which showed that a local temperature minimum would develop between the lower and upper atmospheric regions in conditions of strong irradiation. And this minimum may become unrealistically low when increasingly higher escape rates result from a progressively more intense solar radiation flux. This zero temperature limit, which could not be compensated for by thermal conduction, was called by these authors the energy-limited escape. A simplified equation that corresponds to this energy limit was later widely used by different authors to estimate mass loss from different planets including hot extraterrestrial planets (e.g., Lammer et al. 2003; Lecavelier des Etangs 2007; Hubbard et al. 2007a,b; Davis \& Wheatley 2009).

As shown recently by Muñoz (2007) and Penz et al. (2008a), analytical formulae of Watson et al. (1981) for the "planetary wind" in conditions of strong irradiation can be approximated by the equation

$$
L_{\mathrm{th}} \simeq \frac{r_{\mathrm{EUV}}^{2} F_{\mathrm{EUV}}}{G M_{\mathrm{pl}} / r_{\mathrm{pl}}},
$$

where $r_{\mathrm{EUV}}$ is the altitude where the EUV radiation is absorbed (for hot hydrogen rich gas or ice giants: $r_{\mathrm{EUV}} \approx r_{\mathrm{pl}}$ ) and $r_{\mathrm{pl}}$ and $M_{\mathrm{pl}}$ are the radius and mass of the planet, $F_{\mathrm{EUV}}$ is the stellar EUV radiative energy flux at its orbital distance, and $G$ is Newton's gravitational constant. It indicates also that the limiting effect of conduction on the mass loss rate in the lower thermosphere of an Earth-like planet discovered by Watson et al. (1981) is intrinsically related to the limiting effect of the solar energy deposition in the atmosphere and is a consequence of the latter. It should be noted that Eq. (1) does not depend on thermal conduction and the application of such an equation to hydrogen loss studies is not justified by the derivation of Watson et al. (1981). It is justified by the comparison of hydrodynamic simulations of atmospheric loss from a close-in "Hot Jupiter" orbiting a main sequence G-star and the loss rates estimated with the energylimited equation by Muñoz (2007) and Penz et al. (2008a). That comparison shows that the energy-limited equation, while being much simpler, retains the most essential physics of the hydrodynamic flow and with properly adjusted input parameters gives practically the same loss rates as the hydrodynamic solutions, provided the stellar EUV flux incident on the planet is not more than $\approx 10^{4} \mathrm{erg} \mathrm{cm}^{-2} \mathrm{~s}^{-1}$. So this fact justifies our use of the energy limited equation for the study of the thermal mass loss from hydrogen-rich exoplanets orbiting main sequence $\mathrm{F}, \mathrm{G}, \mathrm{K}$, and M stars.

Furthermore, Yelle (2004), from his hydrodynamic simulations of atmospheric loss from a "Hot Jupiter" as a function of distance from its host star, found that the escape rate varies proportionally to the stellar EUV flux. He then concluded that the escape rate is nearly energy limited, i.e., the escape flux is not determined by the temperature of the atmosphere, but by the amount of stellar EUV energy absorbed in the upper atmosphere.

The basic formula which we use for estimation of the thermal atmospheric loss rate from a close-in exoplanet which includes the effects of strong EUV radiation and strong stellar tidal forces was recently derived by Erkaev et al. (2007). That derivation is based on integration of the mass and energy conservation equations through the thermosphere from the point of minimum temperature to the Roche lobe radius $r_{\mathrm{rl}}$, and does not use any specific assumptions about thermal conduction of atmospheric gas. The resulting equation for the mass loss rate has the following form

$$
L_{\mathrm{th}}=\frac{4 \pi \eta Q}{\Phi_{0} K(\xi)+\frac{v_{\mathrm{rl}}^{2}}{2}+c_{\mathrm{p}}\left(T_{\mathrm{rl}}-T_{0}\right)} .
$$

Here

$\Phi_{0}=G M_{\mathrm{pl}} / r_{\mathrm{pl}}$ 
is the gravitational potential at the planetary surface,

$K(\xi)=1-\frac{3}{2 \xi}+\frac{1}{2 \xi^{3}}<1$

is a non-linear potential energy reduction factor due to the stellar tidal forces, and

$\xi=\frac{r_{\mathrm{rl}}}{r_{\mathrm{pl}}}=d\left(\frac{4 \pi \rho_{\mathrm{pl}}}{9 M_{*}}\right)^{\frac{1}{3}}$

is a dimensionless Roche lobe boundary distance, where $M_{*}$ is the star mass, $\rho_{\mathrm{pl}}$ is planetary density, and $d$ is orbital distance. Also, in Eq. (2), $v_{\mathrm{rl}}$ and $T_{\mathrm{rl}}$ are the flow velocity and temperature at the Roche lobe distance, $r_{\mathrm{rl}} ; T_{0}$ is the minimum temperature at the base of the thermosphere and $c_{\mathrm{p}}$ is the specific heat at constant pressure per unit mass of gas; $\eta$ is the heating efficiency as described in the previous section and $Q$ is the net radiative power per unit solid angle absorbed by the thermosphere, which is

$Q=\int_{r_{\mathrm{pl}}}^{r_{\mathrm{rl}}} q r^{2} \mathrm{~d} r$

where $q$ is the net volume heating rate of the atmosphere. Introducing the average distance $r_{\mathrm{EUV}}$ where the incident stellar radiation energy is deposited in the atmosphere as

$r_{\mathrm{EUV}}=\left(\frac{\int_{r_{\mathrm{pl}}}^{r_{\mathrm{rl}}} q r^{2} \mathrm{~d} r}{F_{\mathrm{EUV}}}\right)^{\frac{1}{2}}$,

and averaging the radiative power $Q$ over the surface of the planet (Erkaev et al. 2007), we obtain the following equation for the thermal loss rate from a "Hot Jupiter"

$L_{\mathrm{th}}=\frac{\pi r_{\mathrm{EUV}}^{2} \eta F_{\mathrm{EUV}}}{\Phi_{0} K(\xi)+\frac{v_{\mathrm{rl}}^{2}}{2}+c_{\mathrm{p}}\left(T_{\mathrm{rl}}-T_{0}\right)}$.

This equation becomes exact under the conditions assumed for its derivation if we know all the appropriate parameters, including the flow velocity and temperature at the Roche lobe distance. But to calculate these values one needs to solve the system of hydrodynamic equations for a flow that transits through a sonic point and becomes supersonic, which is not a simple task. However, for stellar EUV fluxes which are $\lesssim 10^{4} \mathrm{erg} \mathrm{cm}^{-2} \mathrm{~s}^{-1}$, the kinetic and thermal energy terms in the energy balance equation are small when compared to the potential energy term. This conclusion is supported by the full hydrodynamic simulations of Yelle (2004), Muñoz (2007), Penz et al. (2008a), and Murray-Clay et al. (2009). By neglecting these terms in the denominator of Eq. (8), we obtain a modified energy-limited equation for the thermal loss rate

$L_{\mathrm{th}}=\frac{\pi r_{\mathrm{EUV}}^{2} \eta F_{\mathrm{EUV}} \zeta(\xi)}{\Phi_{0}}$

for application to close-in hydrogen-rich "Hot Jupiters". $r_{\mathrm{EUV}}$ is close to the visual planetary radius and can therefore be substituted with $r_{\mathrm{pl}}$ (Yelle 2004). This equation differs from the "standard" energy-limited Eq. (1) by the geometric factor $\pi$, which results from the stellar flux averaging over a planet, the heating efficiency $\eta$ and a loss rate enhancement factor $\zeta$ due to the closeness of the Roche lobe boundary to the planet, which is

$\zeta=\frac{1}{K(\xi)}$.
The above derivation, which does not use any assumptions on thermal conduction in the thermosphere of a planet, clearly demonstrates that this approach to thermal loss calculation is fully applicable not only for terrestrial planets, but also for hot close-in exoplanets provided the heating efficiency by the stellar EUV radiation is appropriately estimated.

These assumptions are comparable to those of Penz et al. (2008b), Penz \& Micela (2008) and Murray-Clay et al. (2009) but differ from those used by Lammer et al. (2003) and Baraffe et al. (2004), who considered the radius $r_{\mathrm{EUV}}$ to be $\approx 3 r_{\mathrm{pl}}$. These early works used a formula for $r_{\text {EUV }}$ derived by Watson et al. (1981) which cannot be applied to "Hot Jupiters" because in those environments conductive cooling is not significant.

Erkaev et al. (2007) showed that Eqs. (9) and (10) can be applied for close-in gas giants at orbital distances $\leq 0.15 \mathrm{AU}$, because due to the Roche lobe effect the upper atmospheres of such planets can also experience hydrodynamic blow-off conditions even if their exobase temperatures are lower than those required for blow-off in the case of the classic Newtonian gravitational potential. For very massive or Jupiter-class exoplanets exposed to less intense stellar EUV fluxes at orbital distances $>0.15 \mathrm{AU}$, the exobase temperatures can be lower than the critical temperature for the onset of the blow-off. This will result in stable upper atmospheres, which experience only Jeans escape.

Because we study the expected mass losses over evolutionary time periods, we have to consider the mass-radius relation. We apply a scaling law which was developed by Lecavelier des Etangs (2007) and fits the mass-radius relation given in Burrows et al. (2000) and Guillot (2005) for exoplanets with $M_{\mathrm{pl}} \gtrsim 0.1 M_{\text {Jup }}$

$r_{\mathrm{pl}}(t)=r_{\infty}\left(1+\beta t^{-0.3}\right)$

with $t$ in Gyr, $\beta=0.2$ for $M_{\mathrm{pl}} \geq 0.3 M_{\mathrm{Jup}}$ and $\beta=0.3$ for $M_{\mathrm{pl}} \approx$ $0.1 M_{\text {Jup }}$. For planets with $M_{\mathrm{pl}}<0.1 M_{\text {Jup }}$ we also used $\beta=0.3$. To study the influence of the heating efficiency on the thermal loss rates we used the values of $\eta$ discussed in Sect. 2.1 and compared the mass loss with $\eta=100 \%$ for the energy limited approach.

Because the age of the majority of the host stars in our exoplanet sample is not well known, we assume that all stars have an average age of about 4 Gyr. We calculated the mass loss rates backwards in time to an initial age of 0.1 Gyr by using Eq. (9) with $M_{\mathrm{pl}}$ and $r_{\mathrm{pl}}$ being the mass and radius obtained from the transiting exoplanets' observations.

\subsection{Stellar EUV evolution with age}

Most previous works scaled the present-day solar EUV luminosity to closer orbital distances or, in evolutionary models, applied the scaling law for the temporal evolution of the stellar radiation for G-type stars derived by Ribas et al. (2005) from the "Sun in Time" program. The "Sun in Time" program is based on a small sample of solar proxies which allow the determination of this average scaling law for the hard X-ray $(\lambda \approx 1-20 \AA)$, soft X-ray $(\mathrm{SXR})(\lambda \approx 20-100 \AA)$ and $\operatorname{EUV}(\lambda \approx 100-1000 \AA)$ radiation.

Penz et al. (2008b) pointed out that stars of the same spectral type show a broad distribution of X-ray luminosity, which varies over a few orders of magnitude. Because of interstellar absorption and the lack of sensitive instruments, this distribution can be observed only for the XUV wavelength in the range of 1-200 $\AA$ ) (e.g., Preibisch \& Feigelson 2005).

Penz et al. (2008b) used ROSAT satellite measurements of G-type stars which cover the transition region between SXR and 


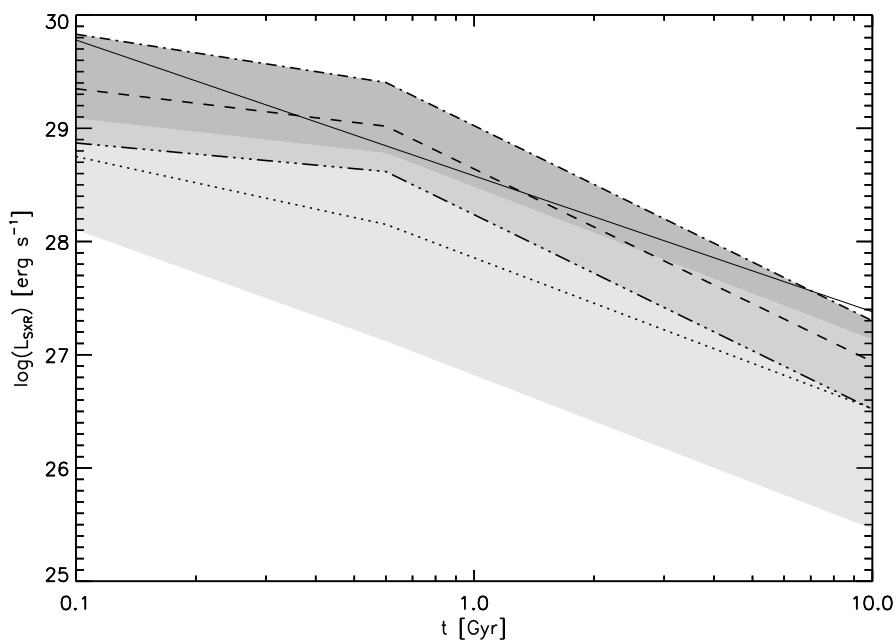

Fig. 1. Average SXR luminosity $L_{\mathrm{SXR}}$ as a function of stellar age for F stars (solid-dotted line), G stars (dashed line), K stars (solid-dotteddotted line), and $\mathrm{M}$ stars (dotted line). The dark grey together with the grey area correspond to the standard deviation $1 \sigma$ of the G-star distribution, and light grey together with the grey area for the $\mathrm{M}$ stars. The grey area is the overlap of both distributions. The thin solid line represents a power law obtained by Ribas et al. (2005) for EUVE satellite data of solar proxies with different ages.

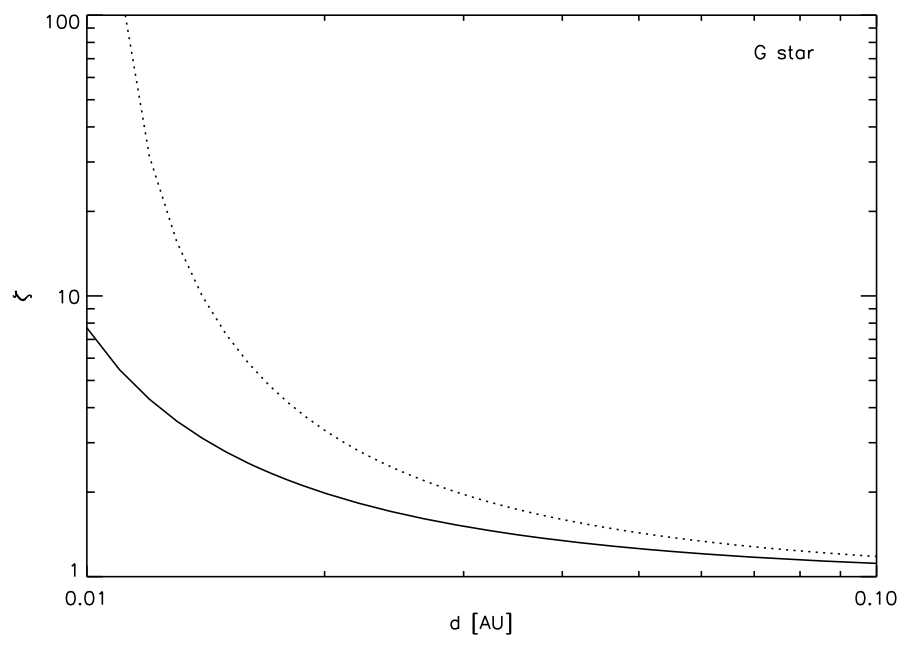

Fig. 2. Roche lobe related mass enhancement factor $\zeta$ as a function of orbital distance for exoplanets with a density of $0.4 \mathrm{~g} \mathrm{~cm}^{-3}$ (dotted line) and $1.3 \mathrm{~g} \mathrm{~cm}^{-3}$ (solid line) around a $\mathrm{G}$ star of one solar mass.

$\operatorname{EUV}(\lambda \approx 100 \AA)$. These data correlate well with the EUVE satellite measurements in the range of $\approx 100-360 \AA$ shown for solar proxies in Ribas et al. (2005). In this study we therefore also use the scaling laws given by Penz et al. (2008b) and Penz \& Micela (2008) for the very extreme UV and X-ray luminosity evolution as a justified EUV proxy. These scaling laws were constructed from the ROSAT satellite observations of stars in the Pleiades and Hyades clusters. The temporal evolution of the stellar SXR flux at the planetary orbits and $r_{\mathrm{pl}}$ are taken into account, while the stellar mass $M_{*}$ and orbital distance $d$ are assumed to be constant. To account for the $\mathrm{F}$ and $\mathrm{K}$ stars in our sample we used the upper limit of $\mathrm{G}$ stars for the F star scaling law and the lower limit for K stars (cf. Penz et al. 2008b, Fig. 2).

For F stars we use

$L_{\mathrm{SXR}}=\left\{\begin{array}{cl}0.284 L_{0} t^{-0.547} & t \leq 0.6 \mathrm{Gyr} \\ 0.155 L_{0} t^{-1.72} & t>0.6 \mathrm{Gyr}\end{array} L_{0}=10^{29.83}\right.$, for G stars

$L_{\mathrm{SXR}}=\left\{\begin{array}{cc}0.375 L_{0} t^{-0.425} & t \leq 0.6 \mathrm{Gyr} \\ 0.19 L_{0} t^{-1.69} & t>0.6 \mathrm{Gyr}\end{array}, L_{0}=10^{29.35}\right.$,

for K stars

$L_{\mathrm{SXR}}=\left\{\begin{array}{cc}0.474 L_{0} t^{-0.324} & t \leq 0.6 \mathrm{Gyr} \\ 0.234 L_{0} t^{-1.72} & t>0.6 \mathrm{Gyr}\end{array}, L_{0}=10^{28.87}\right.$,

and for M stars

$L_{\mathrm{SXR}}=\left\{\begin{array}{ll}0.17 L_{0} t^{-0.77} & t \leq 0.6 \mathrm{Gyr} \\ 0.13 L_{0} t^{-1.34} & t>0.6 \mathrm{Gyr}\end{array}, L_{0}=10^{28.75}\right.$,

with $t$ in Gyr and $L_{\mathrm{SXR}}$ in erg $\mathrm{s}^{-1}$ where $F_{\mathrm{EUV}} \approx L_{\mathrm{SXR}} / 4 \pi d^{2}$. Figure 1 shows the SXR luminosity evolution with time obtained from the scaling laws of F, G, K and M-type stars given in Eqs. (12-15). The solid thin line corresponds to the EUVE power law for solar proxies with different age (Ribas et al. 2005). As one can see, this curve is well within the spread of our scaling law for the SXR G-star distribution.

\section{Results}

Table 2 summarizes the planetary parameters of the 57 transiting exoplanets and the lost mass due to thermal evaporation over 4 Gyr corresponding to heating efficiencies of $10 \%, 25 \%, 60 \%$ and full energy-limited conditions with $\eta=100 \%$. As one can see in Table 2, from 21 F-star transiting exoplanets, only 6 lost $\sim 1-4 \%$ of their initial masses if we consider a heating efficiency of about $10-25 \%$. From our calculations, WASP-12b, which is also the closest of the F-star transit planet sample, experienced the highest mass loss of about $6-12.5 \%$. The same exoplanet also has a low density of about $0.3 \mathrm{~g} \mathrm{~cm}^{-3}$ and the highest Roche lobe related mass loss enhancement factor $\zeta$. If we apply the energy-limited approach with a heating efficiency of $100 \%$, the mass loss would be more than $20 \%$ higher. TRES- $4 \mathrm{~b}$ and WASP$15 \mathrm{~b}$ have the lowest densities of about 0.2 and $0.23 \mathrm{~g} \mathrm{~cm}^{-3}$ at an orbital location of $\approx 0.05 \mathrm{AU}$ and show the next largest mass loss in about $1.3-4 \%$ in our F-star transit exoplanet sample.

One can see from our G star sample that only CoRoT-1b lost more than $1 \%$ of its initial mass over 4 Gyr. CoRoT- $1 b$ lost about $1.3-3 \%$ of its initial mass and is followed by WASP-4 b which lost about $1.1-2.6 \%$ and OGLE-TR-56b with about $0.6-1.46 \%$. All three exoplanets are low density planets and orbit at close distances ofbetween $0.0225-0.0254$ AU. The other gas giants in our G star exoplanet sample have more or less negligible thermal mass loss rates. As expected, the most distant transiting exoplanet HAT80606b at $0.45 \mathrm{AU}$ and a mass of $4 M_{\text {Jup }}$ loses practically no mass.

Compared to the $\mathrm{F}$ and $\mathrm{G}$ star exoplanets, the thermal mass loss of 12 exoplanets in orbits around K-type stars at similar orbital distances around lower mass $\mathrm{K}$ and M-type stars (see Table 2) is negligible. This result is in agreement with Penz \& Micela (2008), because the SXR-ray/EUV flux from cooler stars is significantly lower than the flux from solar-mass stars for a given orbital distance.

As shown in Table 2, our study indicates that the Roche lobe has the greatest influence on the atmospheric mass loss of closein exoplanets. Figs. 2 and 3 show the Roche lobe related mass enhancement factor $\zeta$ as a function of planetary density $\rho$ and orbital distance around G-type stars with $M_{\text {Star }}=1.0 M_{\text {Sun }}$ M-type stars and early $\mathrm{M}$ type stars with $M_{\text {Star }}=0.5 M_{\text {Sun }}$. One can see from Figs. 2 and 3 that close-in exoplanets with low densities 
Table 2. Masses and radii of 57 transiting exoplanets, stellar masses, as well as most of the spectral types (SPT) taken from the Extrasolar Planets Enyclopaedia; status June 2009; http: //www . exoplanet . eu/.

\begin{tabular}{|c|c|c|c|c|c|c|c|c|c|c|c|c|}
\hline & Exoplanet & $\frac{M_{\mathrm{pl}}}{M_{\mathrm{Jup}}}$ & $\frac{r_{\mathrm{pl}}}{r_{\mathrm{Jup}}}$ & $\rho_{\mathrm{pl}}$ & $\frac{M_{\text {star }}}{M_{\text {Sun }}}$ & SPT & $d$ & $\zeta$ & $\frac{\Delta M_{\mathrm{pl}}}{M_{\mathrm{pl}} 10 \%}$ & $\frac{\Delta M_{\mathrm{pl}}}{M_{\mathrm{pl}} 25 \%}$ & $\frac{\Delta M_{\mathrm{pl}}}{M_{\mathrm{pl}} 60 \%}$ & $\frac{\Delta M_{\mathrm{pl}}}{M_{\mathrm{pl}} 100 \%}$ \\
\hline \multirow[t]{26}{*}{$\mathrm{F}$} & & \multicolumn{4}{|c|}{$\left[\mathrm{g} \mathrm{cm}^{-3}\right]$} & \multicolumn{3}{|c|}{$[\mathrm{AU}]$} & \multicolumn{3}{|c|}{ Mass loss [\%] } & \\
\hline & CoRoT-3 b & 21.66 & 1.01 & 26.08 & 1.37 & F3V & 0.057 & 1.08 & 0.0002 & 0.0005 & 0.001 & 0.0017 \\
\hline & CoRoT-4 b & 0.72 & 1.19 & 0.53 & 1.1 & FOV & 0.090 & 1.19 & 0.12 & 0.3 & 0.73 & 1.212 \\
\hline & CoRoT-5 b & 0.46 & 1.28 & 0.27 & 1.00 & F9V & 0.050 & 1.53 & 1.56 & 3.75 & 8.32 & .12 .78 \\
\hline & HAT-P-2 b & 8.62 & 0.95 & 12.43 & 1.29 & F8 & 0.068 & 1.09 & 0.0007 & 0.002 & 0.004 & 0.0069 \\
\hline & HAT-P-4 b & 0.68 & 1.27 & 0.41 & 1.26 & $\mathrm{~F}$ & 0.045 & 1.55 & 0.90 & 2.17 & 5.07 & 8.02 \\
\hline & HAT-P-5 b & 1.06 & 1.26 & 0.66 & 1.16 & $\mathrm{~F}^{\mathrm{x}}$ & 0.041 & 1.48 & 0.42 & 1.02 & 2.43 & 3.95 \\
\hline & HAT-P-6 b & 1.06 & 1.33 & 0.56 & 1.29 & $\mathrm{~F}$ & 0.052 & 1.39 & 0.28 & 0.70 & 1.68 & 2.75 \\
\hline & HAT-P-7 b & 1.77 & 1.36 & 0.87 & 1.47 & $\mathrm{~F}^{\mathrm{x}}$ & 0.038 & 1.53 & 0.23 & 0.56 & 1.34 & 2.21 \\
\hline & HAT-P-8 b & 1.52 & 1.5 & 0.56 & 1.28 & $\mathrm{~F}^{\mathrm{x}}$ & 0.049 & 1.42 & 0.23 & 0.56 & 1.35 & 2.23 \\
\hline & HAT-P-9 b & 0.78 & 1.4 & 0.35 & 1.28 & $\mathrm{~F}$ & 0.053 & 1.48 & 0.63 & 1.52 & 3.58 & 5.76 \\
\hline & OGLE2-TR-L9 b & 4.50 & 1.61 & 1.34 & 1.52 & F3 & 0.041 & 1.39 & 0.05 & 0.11 & 0.27 & 0.44 \\
\hline & OGLE-TR-132 b & 1.14 & 1.18 & 0.86 & 1.26 & $\mathrm{~F}$ & 0.031 & 1.67 & 0.6 & 1.44 & 3.42 & 5.5 \\
\hline & OGLE-TR-182 b & 1.01 & 1.13 & 0.87 & 1.14 & $\mathrm{~F}^{\mathrm{x}}$ & 0.051 & 1.32 & 0.19 & 0.46 & 1.11 & 1.83 \\
\hline & OGLE-TR-211 b & 1.03 & 1.36 & 0.51 & 1.33 & $\mathrm{~F}^{\mathrm{x}}$ & 0.051 & 1.43 & 0.35 & 0.84 & 2.02 & 3.29 \\
\hline & SWEEPS-04 & 3.8 & 0.81 & 8.87 & 1.24 & $\mathrm{~F}^{\mathrm{x}}$ & 0.055 & 1.12 & 0.003 & 0.01 & 0.02 & 0.04 \\
\hline & TrES-4 b & 0.92 & 1.79 & 0.2 & 1.38 & $\mathrm{~F}$ & 0.049 & 1.70 & 1.27 & 3.01 & 6.9 & 10.73 \\
\hline & WASP-1 b & 0.89 & 1.36 & 0.44 & 1.24 & F7V & 0.038 & 1.69 & 1.0 & 2.39 & 5.54 & 8.73 \\
\hline & WASP-12 b & 1.41 & 1.79 & 0.31 & 1.35 & $\mathrm{~F}$ & 0.023 & 3.68 & 5.97 & 12.49 & 23.46 & 31.23 \\
\hline & WASP-14 b & 7.73 & 1.26 & 4.8 & 1.32 & F5V & 0.037 & 1.25 & 0.01 & 0.02 & 0.05 & 0.08 \\
\hline & WASP-3 b & 1.76 & 1.31 & 0.97 & 1.24 & F7V & 0.032 & 1.6 & 0.31 & 0.74 & 1.79 & 2.93 \\
\hline & WASP-7 b & 0.96 & 0.92 & 1.56 & 1.28 & F5V & 0.062 & 1.21 & 0.07 & 0.17 & 0.4 & 0.67 \\
\hline & WASP-13 b & 0.46 & 1.21 & 0.32 & 1.03 & F9 & 0.053 & 1.45 & 1.11 & 2.70 & 6.12 & 9.59 \\
\hline & WASP-15 b & 0.54 & 1.43 & 0.23 & 1.18 & F5 & 0.050 & 1.62 & 1.66 & 3.98 & 8.79 & 13.43 \\
\hline & $\mathrm{X} 0-3 \mathrm{~b}$ & 11.79 & 1.22 & 8.12 & 1.21 & F5V & 0.045 & 1.15 & 0.002 & 0.005 & 0.01 & 0.019 \\
\hline & $\mathrm{X} 0-4 \mathrm{~b}$ & 1.72 & 1.34 & 0.89 & 1.32 & F5V & 0.056 & 1.3 & 0.09 & 0.22 & 0.52 & 0.87 \\
\hline \multicolumn{13}{|c|}{ G } \\
\hline & CoRoT-1 b & 1.03 & 1.49 & 0.37 & 0.95 & G0V & 0.025 & 2.36 & 1.3 & 3.07 & 6.99 & 10.81 \\
\hline & CoRoT-2 b & 3.31 & 1.47 & 1.31 & 0.97 & G & 0.028 & 1.56 & 0.06 & 0.15 & 0.35 & 0.59 \\
\hline & HAT-P-1 b & 0.52 & 1.23 & 0.35 & 1.13 & G0V & 0.055 & 1.43 & 0.32 & 0.78 & 1.88 & 3.07 \\
\hline & HD 149026 b & 0.36 & 0.65 & 1.59 & 1.3 & G0IV & 0.043 & 1.32 & 0.16 & 0.38 & 0.93 & 1.53 \\
\hline & HD 17156 b & 3.21 & 1.02 & 3.72 & 1.24 & G0 & 0.162 & 1.05 & 0.0004 & 0.001 & 0.003 & 0.004 \\
\hline & HD 209458 b & 0.69 & 1.32 & 0.37 & 1.01 & G0V & 0.047 & 1.49 & 0.34 & 0.83 & 1.98 & 3.23 \\
\hline & HD 80606 b & 4.00 & 1.03 & 0.45 & 0.9 & G5 & 0.453 & 1.02 & $3.2 \times 10^{-5}$ & $8.1 \times 10^{-5}$ & 0.0002 & 0.0003 \\
\hline & OGLE-TR-10 b & 0.63 & 1.26 & 0.39 & 1.18 & G & 0.042 & 1.61 & 0.48 & 1.16 & 2.76 & 4.46 \\
\hline & OGLE-TR-56 b & 1.29 & 1.3 & 0.73 & 1.17 & G & 0.023 & 2.2 & 0.6 & 1.46 & 3.45 & 5.53 \\
\hline & SWEEPS-11 & 9.7 & 1.13 & 8.34 & 1.1 & $\mathrm{G}^{\mathrm{x}}$ & 0.030 & 1.24 & 0.002 & 0.005 & 0.01 & 0.02 \\
\hline & TrES-2 & 1.2 & 1.22 & 0.82 & 0.98 & G0V & 0.037 & 1.47 & 0.14 & 0.34 & 0.83 & 1.36 \\
\hline & TrES-3 & 1.92 & 1.3 & 1.1 & 0.92 & G & 0.023 & 1.81 & 0.22 & 0.52 & 1.26 & 2.08 \\
\hline & WASP-4 b & 1.12 & 1.42 & 0.49 & 0.9 & G7V & 0.023 & 2.32 & 1.09 & 2.60 & 5.97 & 9.33 \\
\hline & WASP-5 b & 1.58 & 1.09 & 1.51 & 0.97 & G4V & 0.027 & 1.55 & 0.11 & 0.28 & 0.68 & 1.12 \\
\hline & WASP-6 b & 0.5 & 1.22 & 0.34 & 0.88 & G8 & 0.042 & 1.57 & 0.66 & 1.60 & 3.76 & 6.04 \\
\hline & WASP-8 b & 2.23 & 1.17 & 1.73 & 1.00 & G6 & 0.079 & 1.13 & 0.006 & 0.014 & 0.034 & 0.057 \\
\hline & WASP-9 b & 2.30 & 1.30 & 1.30 & 1.01 & G0 & 0.031 & 1.49 & 0.07 & 0.16 & 0.39 & 0.65 \\
\hline & $\mathrm{X} 0-1 \mathrm{~b}$ & 0.9 & 1.18 & 0.67 & 1 & G1V & 0.049 & 1.35 & 0.12 & 0.29 & 0.69 & 1.15 \\
\hline & $\mathrm{X} 0-5 \mathrm{~b}$ & 1.08 & 1.09 & 1.04 & 0.88 & G8V & 0.049 & 1.28 & 0.06 & 0.15 & 0.36 & 0.59 \\
\hline \multicolumn{13}{|c|}{$\mathrm{K}$} \\
\hline & HAT-P-11 b & 0.08 & 0.42 & 1.34 & 0.81 & K4 & 0.053 & 1.22 & 0.16 & 0.53 & 0.97 & 1.59 \\
\hline & HAT-P-3 b & 0.6 & 0.89 & 1.05 & 0.94 & K & 0.039 & 1.38 & 0.07 & 0.17 & 0.41 & 0.68 \\
\hline & HAT-P-12 b & 0.21 & 0.96 & 0.30 & 0.73 & $\mathrm{~K}^{\mathrm{x}}$ & 0.038 & 1.63 & 1.01 & 2.46 & 5.60 & 8.82 \\
\hline & HD 189733 b & 1.13 & 1.14 & 0.95 & 0.8 & $\mathrm{~K} 1-\mathrm{K} 2$ & 0.031 & 1.51 & 0.07 & 0.18 & 0.42 & 0.70 \\
\hline & Lupus-TR-3 b & 0.81 & 0.89 & 1.43 & 0.87 & K1V & 0.046 & 1.26 & 0.02 & 0.06 & 0.15 & 0.24 \\
\hline & OGLE-TR-111 b & 0.53 & 1.07 & 0.54 & 0.82 & K & 0.047 & 1.37 & 0.10 & 0.26 & 0.62 & 1.02 \\
\hline & OGLE-TR-113 b & 1.32 & 1.09 & 1.27 & 0.78 & $\mathrm{~K}$ & 0.023 & 1.69 & 0.10 & 0.23 & 0.56 & 0.93 \\
\hline & TrES-1 & 0.61 & 1.08 & 0.6 & 0.87 & $\mathrm{~K} 0 \mathrm{~V}$ & 0.039 & 1.48 & 0.13 & 0.32 & 0.76 & 1.26 \\
\hline & WASP-10 b & 3.06 & 1.08 & 3.01 & 0.71 & K5 & 0.037 & 1.23 & 0.01 & 0.01 & 0.03 & 0.05 \\
\hline & WASP-11 b & 0.46 & 1.05 & 0.5 & 0.82 & $\mathrm{~K} 3 \mathrm{~V}$ & 0.044 & 1.43 & 0.15 & 0.38 & 0.91 & 1.51 \\
\hline & WASP-2 b & 0.88 & 1.02 & 1.04 & 0.84 & $\mathrm{~K} 1 \mathrm{~V}$ & 0.031 & 1.5 & 0.08 & 0.21 & 0.49 & 0.82 \\
\hline & $\mathrm{X} 0-2 \mathrm{~b}$ & 0.57 & 0.97 & 0.77 & 0.98 & K0V & 0.037 & 1.48 & 0.12 & 0.30 & 0.71 & 1.18 \\
\hline \multicolumn{13}{|c|}{ M } \\
\hline & GJ 436 b & 0.07 & 0.44 & 1.06 & 0.45 & $\mathrm{M} 2.5$ & 0.029 & 1.41 & 0.43 & 1.41 & 2.51 & 4.08 \\
\hline
\end{tabular}

Spectral types that are roughly estimated corresponding to the mass sequence are indicated with a superscript $x$. The lost mass is calculated over a period of $4 \mathrm{Gyr}$ for three different values of heating efficiency $(10 \%, 25 \%, 60 \%, 100 \%)$. The planetary densities and the Roche lobe related mass loss enhancement factor $\zeta$ are also given. 


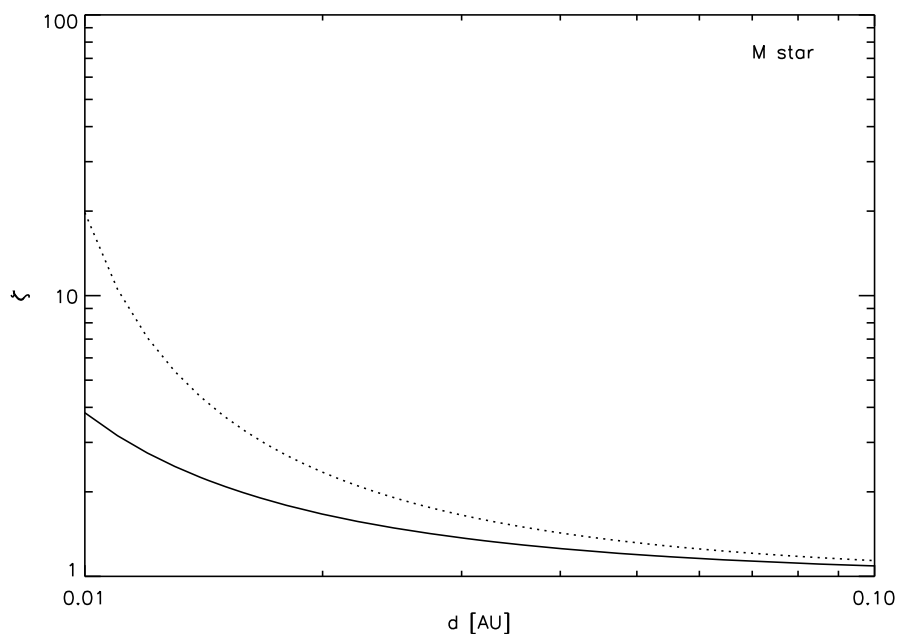

Fig. 3. Roche lobe related mass enhancement factor $\zeta$ as a function of orbital distance for exoplanets with a density of $0.4 \mathrm{~g} \mathrm{~cm}^{-3}$ (dotted line) and $1.3 \mathrm{~g} \mathrm{~cm}^{-3}$ (solid line) around a M star with a mass of $0.5 M_{\text {Sun }}$.

are more affected than similar planets with a higher density. Our study indicates also that the Roche lobe effect on a planet at a similar orbital distance is more efficient for larger and more massive stars compared to smaller ones with lower masses. Figures 2 and 3 also show that the Roche lobe effect dramatically enhances the mass loss at orbital distances $\leq 0.02$ AU. Exoplanets with densities which are $\leq 0.55 \mathrm{~g} \mathrm{~cm}^{-3}$ can lose their initial hydrogen inventory if they orbit around G-type stars at distances of about 0.01 AU. Baraffe et al. (2004) compared the ratio of the mass loss timescale $t_{\dot{\mathrm{M}}}$ to the thermal timescale $t_{\mathrm{th}}$, which is characterized by the Kelvin-Helmholtz timescale and found that, when $t_{\dot{\mathrm{M}}} / t_{\text {th }}$ becomes $<1$, the evolution of "Hot Jupiters" changes drastically, which results in rapid expansion of the planetary radius and enhances the mass loss. Our model results indicate that close-in hydrogen-rich gas giants with low densities at orbital distances $\leq 0.015$ AU may experience this violent mass loss effect.

Table 3 shows the integrated mass loss over 4 Gyr for gas giants with masses of 1.0 and $0.5 M_{\text {Jup }}$ and a "Hot Neptune" $\left(M_{\mathrm{pl}}=0.054 M_{\mathrm{Jup}}\right)$ and densities of 0.4 and $1.241 \mathrm{~g} \mathrm{~cm}^{-3}$ at orbital distances of between 0.01-0.025 AU around a Sun-like $\operatorname{star}\left(M_{\text {Star }}=1.0 M_{\text {Sun }}\right)$ and a $\mathrm{K}$ type star with $M_{\text {Star }}=0.8 M_{\text {Sun }}$ for various heating efficiencies. One can see from Table 3 that thermal evaporation may be an efficient loss process which can efficiently evaporate the dense hydrogen envelopes of close-in exoplanets in the Neptune-mass domain at orbital distances $d<$ $0.02 \mathrm{AU}$ around G-type stars. Jupiter-mass exoplanets with high densities $\left(>1 \mathrm{~g} \mathrm{~cm}^{-3}\right)$ experience negligible thermal atmospheric mass loss at orbital distances beyond $\geq 0.015$ AU. Between $0.01-$ $0.015 \mathrm{AU}$, similar gas giants may lose $\leq 10 \%$ of their initial atmosphere. Low density gas giants can lose a significant fraction of their hydrogen atmospheres at $\approx 0.01 \mathrm{AU}$. Gas giants with masses of $\sim 0.5 M_{\text {Jup }}$ and densities of about $1.25 \mathrm{~g} \mathrm{~cm}^{-3}$ which orbit G-stars can lose about 6-17\% of their mass at orbital distances of about $0.01 \mathrm{AU}$. We note also that the majority of the thermal mass loss occurs during the first Gyr when the EUV radiation of a young star is much higher compared to ages older than 1 Gyr. If we consider that Lyman- $\alpha$ cooling becomes dominant for EUV fluxes $\geq 10^{4} \mathrm{erg} \mathrm{cm}^{-2}$, we can see that the mass loss will be lower and comparable to $10 \%$ of $\eta$ cases at distances $<0.017 \mathrm{AU}$. The same exoplanets experience less mass loss if their orbits are located at similar distances around K-type stars. One can also see from Table 3 that the recently discovered transiting "Super-Earth" CoRoT-7b (Léger et al. 2009), which is orbiting a $\mathrm{K}$ star at about $0.017 \mathrm{AU}$, cannot be the remaining core of an evaporated "Hot Jupiter" or even lower mass hydrogen-rich gas giant. For instance, a low density $\left(\rho_{\mathrm{pl}} \sim 0.4 \mathrm{~g} \mathrm{~cm}^{-3}\right)$ gas giant around a $\mathrm{K}$ star at an orbital distance of about $0.017 \mathrm{AU}$ and a mass of about $0.5 M_{\text {Jup }}$ with a heating efficiency of 10-25\% loses $\sim 1.35-3.45 \%$ of its initial mass after 4 Gyr. Even if one assumes energy-limited loss with $\eta=100 \%$, the same planet would lose about $16 \%$ of its mass. On the other hand, a "Hot sub-Neptune" with a low density at the same orbital distance could lose its hydrogen envelope so that only the planet's core remains. We cannot, therefore, rule out that CoRoT-7b is a remnant of a thermally evaporated and non-thermally eroded "hot sub-Neptune-mass" object.

Figure 4 shows the effect of thermal mass loss from two gas giants with an initial mass of $0.5 M_{\text {Jup }}$ and densities of $\rho=$ $1.24 \mathrm{~g} \mathrm{~cm}^{-3}$ and $\rho=0.4 \mathrm{~g} \mathrm{~cm}^{-3}$ at orbital distances $\leq 0.025 \mathrm{AU}$ for $\eta$ of 10 and $25 \%$ in an area where so far no Jupiter-mass or sub-Jupiter-class exoplanets have been discovered. The mass evolution of the test planets is calculated over 4 Gyr for both densities and heating efficiencies. As one can see, thermal atmospheric mass loss is not efficient enough to evaporate gas giants which may be located in this area down to their core sizes. This result is contrary to the findings of the recent study of Davis \& Wheatley (2009) who probably have overestimated the thermal mass loss rates using the energy-limited equation but neglect accurate heating efficiencies and thermospheric cooling processes. If this deficiency of discovered exoplanets is related to the mass loss, then non-thermal processes have to be responsible for it. On the other hand, one can see in Fig. 4 that due to the Roche lobe effect, which is independent of $\eta$, hydrogen-rich low density gas giants within the mass range of HAT-P-1b, HAT-P-4b, HAT-P9b, CoRoT-5b, WASP-6b, WASP-11b, WASP-13b, WASP-15b, and Saturn should evaporate down to their coresize at distances $<0.013 \mathrm{AU}$. In the following section we investigate whether stellar wind and CME plasma-induced non-thermal atmospheric erosion can effectively modify the mass evolution of non- or weakly magnetized gas giants at orbital distances $\leq 0.1 \mathrm{AU}$.

\section{Atmospheric mass loss related to stellar wind and CME induced ion erosion}

Taking into account that tidal-locking of short periodic exoplanets may result in weaker intrinsic magnetic moments, as compared to fast rotating Jupiter-class exoplanets at larger orbital distances (Grießmeier et al. 2004), Khodachenko et al. (2007) found that the encountering of dense stellar wind and CME plasma may compress the magnetosphere and force the magnetospheric standoff location to move down to the heights where ionization and ion pick-up of a planetary neutral atmosphere by the CME plasma flow takes place.

In our Solar System, it is known that magnetosphere-like structures are found on all planets regardless of whether the planet has an intrinsic global magnetic field or not. The solar wind interaction with a planet produces a magnetosphere-like structure near the planet with common features such as a bow shock, magnetosheath, magnetotail, and boundary layers. In the case of intrinsically magnetized planets like the Earth, a magnetosphere is formed to resist the oncoming solar wind.

For a planet like Venus or Mars, which has no global intrinsic magnetic field but has an atmosphere, an induced magnetosphere is created by the magnetized solar wind interaction with the highly conducting ionosphere. The induced magnetosphere 
Table 3. The mass loss from "Hot Jupiters" and "Hot Neptunes" with low and high densities at four different orbital distances around a G star $\left(1 M_{\text {Sun }}\right)$ and a $\mathrm{K} \operatorname{star}\left(0.8 M_{\text {Sun }}\right)$.

\begin{tabular}{|c|c|c|c|c|c|c|c|c|c|}
\hline & $\frac{M_{\mathrm{pl}}}{M_{\mathrm{Jup}}}$ & $\frac{\rho_{\mathrm{pl}}}{\rho_{\mathrm{Jup}}}$ & $\frac{\Delta M_{\mathrm{pl}}}{M_{\mathrm{pl}}^{100 \%}}$ & $\frac{\Delta M_{\mathrm{pl}}}{M_{\mathrm{pl}}^{25 / 6}}$ & $\frac{\Delta M_{\mathrm{pl}}}{M_{\mathrm{pl}}^{600 \%}}$ & $\frac{\Delta M_{\mathrm{pl}}}{M_{\mathrm{pl}}^{100 \%}}$ & $\frac{\Delta M_{\mathrm{pl}}}{M_{\mathrm{pl}}^{10 \% / 6-60 \%}}$ & $\frac{\Delta M_{\mathrm{pl}}}{M_{\mathrm{pl}}^{100 / \sigma 25 \%}}$ & $\zeta$ \\
\hline $0.01 \mathrm{AU}, \mathrm{G}$ star & \multicolumn{9}{|c|}{ Mass loss [\%] } \\
\hline & 1 & 1.24 & 2.95 & 7.74 & 21.59 & 52.42 & 2.95 & 2.95 & 8.24 \\
\hline & 0.5 & 1.24 & 6.09 & 17.12 & - & - & 6.09 & 6.09 & 8.24 \\
\hline & 0.054 & 1.61 & 38.17 & - & - & - & 38.17 & 38.17 & 5.90 \\
\hline & 1 & 0.4 & 77.43 & - & - & - & 77.43 & 77.43 & $>100$ \\
\hline & 0.5 & 0.4 & - & - & - & - & - & - & $>100$ \\
\hline & 0.054 & 0.4 & - & - & - & - & - & - & $>100$ \\
\hline \multicolumn{10}{|l|}{$0.017 \mathrm{AU}, \mathrm{G}$ star } \\
\hline & 1 & 1.24 & 0.38 & 0.94 & 2.28 & 3.84 & 0.45 & 0.40 & 2.40 \\
\hline & 0.5 & 1.24 & 0.75 & 1.90 & 4.64 & 7.90 & 0.90 & 0.80 & 2.40 \\
\hline & 0.054 & 1.61 & 4.40 & 11.54 & 32.03 & 87.08 & 5.21 & 4.64 & 2.17 \\
\hline & 1 & 0.4 & 2.08 & 5.34 & 13.80 & 25.71 & 2.46 & 2.19 & 4.82 \\
\hline & 0.5 & 0.4 & 4.23 & 11.24 & 33.23 & - & 5.02 & 4.47 & 4.82 \\
\hline & 0.054 & 0.4 & 51.47 & - & - & - & - & 56.79 & 4.82 \\
\hline \multicolumn{10}{|l|}{$0.020 \mathrm{AU}, \mathrm{G}$ star } \\
\hline & 1 & 1.24 & 0.23 & 0.59 & 1.42 & 2.37 & 0.31 & 0.26 & 2.02 \\
\hline & 0.5 & 1.24 & 0.47 & 1.18 & 2.86 & 4.82 & 0.62 & 0.52 & 2.02 \\
\hline & 0.054 & 1.61 & 2.79 & 7.16 & 18.48 & 34.41 & 3.62 & 3.04 & 1.87 \\
\hline & 1 & 0.4 & 1.11 & 2.80 & 6.93 & 12.02 & 1.45 & 1.21 & 3.32 \\
\hline & 0.5 & 0.4 & 2.23 & 5.73 & 14.74 & 27.26 & 2.93 & 2.44 & 3.32 \\
\hline & 0.054 & 0.4 & 20.29 & - & - & - & 27.06 & 22.23 & 3.32 \\
\hline \multicolumn{10}{|l|}{$0.025 \mathrm{AU}, \mathrm{G}$ star } \\
\hline & 1 & 1.24 & 0.13 & 0.32 & 0.78 & 1.30 & 0.20 & 0.15 & 1.69 \\
\hline & 0.5 & 1.24 & 0.26 & 0.65 & 1.56 & 2.62 & 0.40 & 0.30 & 1.69 \\
\hline & 0.054 & 1.61 & 1.56 & 3.96 & 9.84 & 17.16 & 2.34 & 1.79 & 1.60 \\
\hline & 1 & 0.4 & 0.53 & 1.34 & 3.26 & 5.52 & 0.82 & 0.62 & 2.37 \\
\hline & 0.5 & 0.4 & 1.07 & 2.71 & 6.68 & 11.51 & 1.64 & 1.24 & 2.37 \\
\hline & 0.054 & 0.4 & 9.08 & 25.53 & - & - & 13.71 & 10.44 & 2.37 \\
\hline $0.01 \mathrm{AU}, \mathrm{K}$ star & \multicolumn{9}{|c|}{ Mass loss [\%] } \\
\hline & 1 & 1.24 & 0.88 & 2.23 & 5.51 & 9.54 & 1.01 & 0.92 & 6.16 \\
\hline & 0.5 & 1.24 & 1.77 & 4.55 & 11.69 & 21.46 & 2.03 & 1.85 & 6.16 \\
\hline & 0.054 & 1.61 & 9.17 & 26.80 & - & - & 10.41 & 9.54 & 4.71 \\
\hline & 1 & 0.4 & 76.58 & 87.37 & - & - & 80.24 & 77.83 & 247 \\
\hline & 0.5 & 0.4 & 84.14 & - & - & - & 87.83 & 85.37 & 247 \\
\hline & 0.054 & 0.4 & - & - & - & - & - & - & 247 \\
\hline \multicolumn{10}{|l|}{$0.017 \mathrm{AU}, \mathrm{K}$ star } \\
\hline & 1 & 1.24 & 0.13 & 0.33 & 0.80 & 1.34 & 0.22 & 0.16 & 2.20 \\
\hline & 0.5 & 1.24 & 0.27 & 0.67 & 1.61 & 2.71 & 0.43 & 0.32 & 2.20 \\
\hline & 0.054 & 1.61 & 1.55 & 3.93 & 9.80 & 17.15 & 2.44 & 1.81 & 2.02 \\
\hline & 1 & 0.4 & 0.67 & 1.70 & 4.15 & 7.09 & 1.07 & 0.79 & 3.99 \\
\hline & 0.5 & 0.4 & 1.35 & 3.44 & 8.61 & 15.16 & 2.16 & 1.59 & 3.99 \\
\hline & 0.054 & 0.4 & 11.47 & 35.21 & - & - & 18.23 & 13.41 & 3.99 \\
\hline \multicolumn{10}{|l|}{$0.020 \mathrm{AU}, \mathrm{K}$ star } \\
\hline & 1 & 1.24 & 0.09 & 0.21 & 0.51 & 0.85 & 0.16 & 0.11 & 1.89 \\
\hline & 0.5 & 1.24 & 0.17 & 0.42 & 1.02 & 1.71 & 0.31 & 0.21 & 1.89 \\
\hline & 0.054 & 1.61 & 1.00 & 2.53 & 6.21 & 10.63 & 1.78 & 1.23 & 1.77 \\
\hline & 1 & 0.4 & 0.38 & 0.95 & 2.30 & 3.87 & 0.68 & 0.47 & 2.92 \\
\hline & 0.5 & 0.4 & 0.76 & 1.91 & 4.67 & 7.98 & 1.37 & 0.94 & 2.92 \\
\hline & 0.054 & 0.4 & 6.24 & 16.87 & 56.87 & - & 11.07 & 7.65 & 2.92 \\
\hline \multicolumn{10}{|l|}{$0.025 \mathrm{AU}, \mathrm{K}$ star } \\
\hline & 1 & 1.24 & 0.05 & 0.12 & 0.29 & 0.48 & 0.10 & 0.06 & 1.62 \\
\hline & 0.5 & 1.24 & 0.10 & 0.24 & 0.57 & 0.95 & 0.21 & 0.13 & 1.62 \\
\hline & 0.054 & 1.61 & 0.57 & 1.44 & 3.49 & 5.90 & 1.20 & 0.76 & 1.54 \\
\hline & 1 & 0.4 & 0.19 & 0.48 & 1.15 & 1.92 & 0.41 & 0.26 & 2.18 \\
\hline & 0.5 & 0.4 & 0.38 & 0.95 & 2.31 & 3.88 & 0.82 & 0.51 & 2.18 \\
\hline & 0.054 & 0.4 & 3.10 & 8.00 & 20.99 & 40.46 & 6.52 & 4.11 & 2.18 \\
\hline
\end{tabular}

The superscripts $10 \%-25 \%$ and $10 \%-60 \%$ correspond to a time depended $\eta$ which has a low heating efficiency of $10 \%$ if the EUV flux is $\geq 10^{4} \mathrm{erg} \mathrm{cm}^{-2} \mathrm{~s}^{-1}$ and $25 \%$ and $60 \%$ for EUV flux values $<10^{4} \mathrm{erg} \mathrm{cm}^{-2} \mathrm{~s}^{-1}$ (Murray-Clay et al. 2009). 


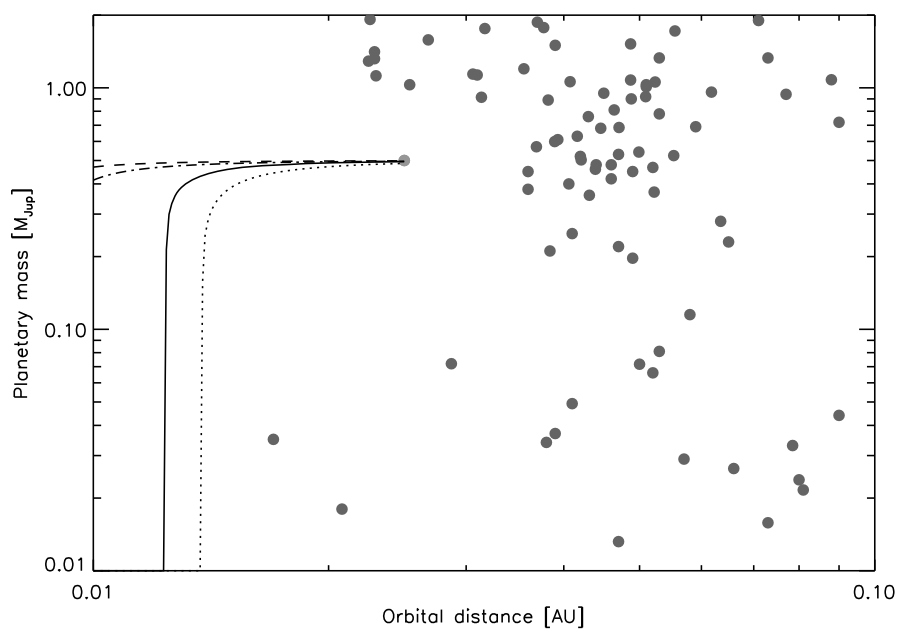

Fig. 4. Final masses corresponding to thermal mass loss integrated over 4 Gyr for high $\left(\rho=1.24 \mathrm{~g} \mathrm{~cm}^{-3}\right)$ and low $\left(\rho=0.4 \mathrm{~g} \mathrm{~cm}^{-3}\right)$ density gas giants with initial masses of $0.5 M_{\text {Jup }}$. Dashed line: $\rho=1.24 \mathrm{~g} \mathrm{~cm}^{-3}$, $\eta=10 \%$; dashed-dotted line: $\rho=1.24 \mathrm{~g} \mathrm{~cm}^{-3}, \eta=25 \%$; solid line: $\rho=0.4 \mathrm{~g} \mathrm{~cm}^{-3}, \eta=10 \%$; dotted line: $\rho=0.4 \mathrm{~g} \mathrm{~cm}^{-3}, \eta=25 \%$. The filled circles show discovered exoplanets in a mass range between $0.01-1.0 M_{\text {Jup }}$ and at orbital distances between 0.01-0.1 AU.

is therefore analogous to the magnetosphere of an intrinsically magnetized planet, but occupies a smaller volume (Zhang et al. 2007). We note that in the case of Mars or Venus, the ionospheric pressure is mostly larger than or comparable to the solar wind ram pressure, both at solar wind maximum and minimum. The interaction of the post-shock solar wind flow with the ionosphere results in a distinct boundary. This is the so-called ionopause which confines the thermal plasma of the ionosphere and marks its top boundary (cf., Phillips \& McComas 1991).

Whether the stellar wind is stopped well above the planet depends on the pressure balance between the stellar wind ram pressure $\left(p_{\mathrm{sw}}=\rho_{\mathrm{sw}, \mathrm{CME}} v_{\mathrm{sw}, \mathrm{CME}}^{2}\right)$ and the maximum ionospheric thermal pressure $\left(p_{\text {ion }}=n_{\mathrm{i}} k\left(T_{\mathrm{e}}+T_{\mathrm{i}}\right)\right)$, possible induced magnetic pressure and neutral atmospheric pressure, where the ionospheric thermal pressure is the main contributor in the upper atmosphere. To first order, here we simply compare the stellar wind ram pressure with the peak ionospheric pressure to determine the height where the stellar wind would be stationary. It is reasonable to postulate that the stellar wind will be absorbed by the planetary atmosphere if the ram pressure is larger than the ionospheric pressure. Such an extreme plasma-atmosphere interaction could result in a large atmospheric mass loss due to ion pick-up and other non-thermal escape processes.

Thus, by using this analogy we can expect that the ionosphere of non- or weakly magnetized "Hot Jupiters" represents an obstacle similar to that where a magnetosphere is present. The difference between the ionized and the magnetic obstacle may be in their distance above the visual radius of the planet.

Figure 5 illustrates the stellar plasma interaction with non- or weakly magnetized gas giants. The dashed line corresponds to a critical planetary obstacle $\left(\mathrm{IP}_{\mathrm{c}}\right)$ deeper inside the atmosphere $\left(P_{\text {tot }}>10^{-4}\right.$ dyn $\left.\mathrm{cm}^{-2}\right)$ but above the visual radii, where one can assume that the $\mathrm{H}^{+}$ion loss rate for a Jupiter-type gas giant should be very large. For higher planetary obstacle locations where the pick-up ion loss becomes comparable to the thermal loss rate from a "Hot Jupiter", the $\mathrm{H}^{+}$loss rate is negligible when integrated over the lifetime of the planet (Khodachenko et al. 2007). If the stellar plasma flow interacts below this critical boundary, the mass loss rate from a "Hot Jupiter" $\left(M_{\mathrm{pl}} \leqslant M_{\mathrm{Jup}}\right)$

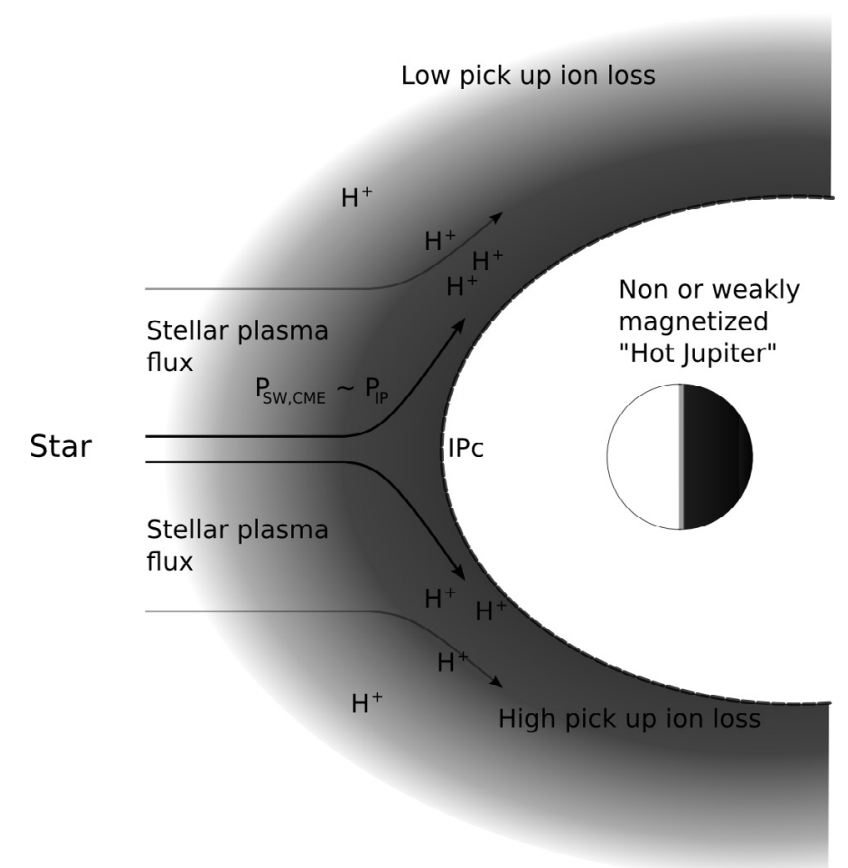

Fig. 5. Illustration of the stellar plasma interaction with non- or weakly magnetized gas giants.

becomes larger and may erode the planet's hydrogen inventory (Khodachenko et al. 2007).

Thus, from the study of Khodachenko et al. (2007), we know that ion pick-up loss is negligible for "Hot Jupiters" if a planetary obstacle builds up at total atmospheric pressures $\leq 10^{-4} \mathrm{dyn} \mathrm{cm}^{-2}$ (shadowed area in Fig. 5). To compare the stellar wind and CME ram pressure with the ionospheric pressure of "Hot Jupiters", one has to know the stellar wind and CME plasma density and velocity as a function of orbital distance, as well as the ionospheric density profile, and the ion temperature and electron temperature of a"Hot Jupiter".

By considering that the Sun is a typical G-type star, we assume a similarity of the basic parameters and their spatial behavior for the stellar wind off other G-type stars and those in the solar wind. In view of the limited amount of precise quantitative information regarding the stellar (non-solar) winds, such a solar-stellar analogy principle is widely used for the investigation of basic processes of the stellar wind - planet interactions. Therefore, in the present study we use the Sun as a proxy in estimating the stellar wind and CME density and speed dependence as a function of the orbital distance $d$. In particular, for the approximation of the stellar wind density we use the empirical power-law formula

$$
\begin{aligned}
n_{\mathrm{sw}}=\left(1.07\left(\frac{d}{r_{\text {Sun }}}\right)^{-3.1}+19.94\right. & \left(\frac{d}{r_{\text {Sun }}}\right)^{-6.06} \\
& \left.+22.10\left(\frac{d}{r_{\text {Sun }}}\right)^{-12.93}\right) \times 10^{7}
\end{aligned}
$$

which was derived from Skylab coronograph observations (Guhathakurta et al. 1996). The stellar wind and CME speed can be approximated by the formula

$v_{\mathrm{sw}}^{2}=v_{0}^{2} \cdot\left(1-\mathrm{e}^{(2.8-d) / 8.1}\right)$

developed by Sheeley et al. (1997) on the basis of tracking several solar wind density enhancements at close distances $(d<$ $0.1 \mathrm{AU})$. For the stellar wind speed, one can take in this formula 

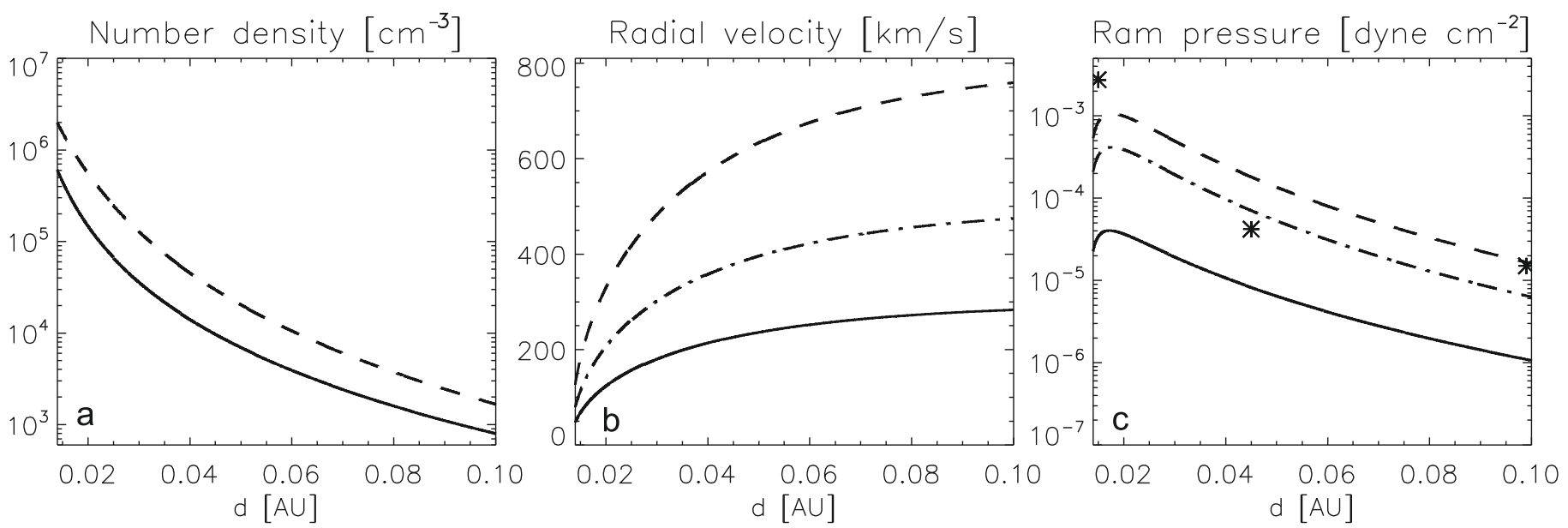

Fig. 6. Stellar wind and CME parameters versus orbital distance corresponding to a Sun-like G star. a) density: stellar wind (solid line), CME (dashed line); b) radial velocity: stellar wind (solid line), fast CME (dashed line), average CME (dashed-dotted line); c) ram pressure: stellar wind (solid line), fast CME (dashed line), average CME (dashed-dotted line). The asterisks correspond to the ion pressure modeled by Yelle (2004) at $2.3 r_{\mathrm{pl}}$ above the planetary radius.

Table 4. Stellar wind and planetary plasma parameters.

\begin{tabular}{lccccccc}
\hline \hline$d[\mathrm{AU}]$ & $n_{\mathrm{sw}}\left[\mathrm{cm}^{-3}\right]$ & $v_{\mathrm{sw}}\left[\mathrm{km} \mathrm{s}^{-1}\right]$ & $n_{\mathrm{CME}}\left[\mathrm{cm}^{-3}\right]$ & $v_{\mathrm{CME}}^{a v}\left[\mathrm{~km} \mathrm{~s}^{-1}\right]$ & $v_{\mathrm{CME}}^{\text {fast }}\left[\mathrm{km} \mathrm{s}^{-1}\right]$ & $n_{\mathrm{H}^{+}}\left[\mathrm{cm}^{-3}\right]$ & $T_{\mathrm{i}}+T \mathrm{e}[\mathrm{K}]$ \\
\hline 0.1 & 794 & 283 & 1667 & 474 & 759 & $6 \times 10^{6}$ & $2.0 \times 10^{4}$ \\
0.045 & 9625 & 226 & $2.95 \times 10^{4}$ & 378 & 605 & $1 \times 10^{7}$ & $3.0 \times 10^{4}$ \\
0.015 & $4.48 \times 10^{5}$ & 68 & $1.54 \times 10^{6}$ & 113 & 181 & $4 \times 10^{8}$ & $5.0 \times 10^{4}$ \\
\hline
\end{tabular}

Table 5. Dynamic stellar plasmas and planetary ion pressures estimated for different orbital locations.

\begin{tabular}{lcccc}
\hline \hline$d[\mathrm{AU}]$ & $P_{\mathrm{sw}}\left[10^{-7} \mathrm{dyn} \mathrm{\textrm {cm } ^ { - 2 } ]}\right.$ & $P_{\mathrm{CME}}^{a v}\left[10^{-7} \mathrm{dyn} \mathrm{cm}^{-2}\right]$ & $P_{\mathrm{CME}}^{\text {fast }}\left[10^{-7}{\left.\mathrm{dyn} \mathrm{cm}^{-2}\right]}\right.$ & $P_{\text {Ion }}\left[10^{-7} \mathrm{dyn} \mathrm{cm}^{-2}\right]$ \\
\hline 0.1 & 11.0 & 63.0 & 160.0 & 140.0 \\
0.045 & 82.0 & 705.0 & 1805.0 & 305.0 \\
0.015 & 341.0 & 3294.0 & 8432.0 & $4.0 \times 10^{4}$ \\
\hline
\end{tabular}

$v_{0}=300 \mathrm{~km} \mathrm{~s}^{-1}$, whereas for average and fast CMEs one can use $v_{0}=500 \mathrm{~km} \mathrm{~s}^{-1}$ and $800 \mathrm{~km} \mathrm{~s}^{-1}$. The decrease of the average CME plasma density $n_{\mathrm{CME}}$ at orbital distances $d \leq 0.1 \mathrm{AU}$ is described by the formula $n_{\mathrm{CME}}(d)=n_{0}\left(d / d_{0}\right)^{-3.6}$, which for $n_{0}=2 \times 10^{6} \mathrm{~cm}^{-3}$ and $d_{0}=3 r_{\text {Sun }}$ gives a good approximation for the values estimated from the SOHO/LASCO coronograph images (Lara et al. 2004). These power-law approximations of the CME density follow from the analysis of solar CMEassociated brightness enhancements in white-light coronagraph images (e.g., Khodachenko 2007, and references therein). The average mass of CMEs is $\approx 10^{15} \mathrm{~g}$ and the average duration of CMEs, at distances $(6 \ldots 10) r_{\text {Sun }}$ is $\approx 8 \mathrm{~h}$.

Figure 6 shows the resulting stellar wind and CME plasma parameters as well as the corresponding ram pressures. Table 4 summarizes particular values of the stellar wind and CME plasma parameters obtained from these approximations for the given orbital distances $0.015 \mathrm{AU}, 0.045 \mathrm{AU}$ and $0.1 \mathrm{AU}$. To estimate the ion pressure for assumed non- or weakly magnetized "Hot Jupiters" we use the $\mathrm{H}^{+}$ion profiles and temperatures modelled by Yelle (2004) and assumed, as did this author, that under extreme conditions $T_{\mathrm{i}}=T_{\mathrm{e}}=T_{\mathrm{n}}$. Yelle (2004) applied a coupled photochemical and hydrodynamic model for hydrogen-rich atmospheres of "Hot Jupiters" in orbits with semi-major axes from 0.01 to $0.1 \mathrm{AU}$. The modelled planetary plasma parameters correspond to planetocentric distances of about $3.3 r_{\mathrm{pl}}$ and are shown in Table 5. We note that the model of Yelle (2004) does not include the interaction with the incoming stellar wind, which was recently treated by Murray-Clay et al. (2009) for a Hot Jupiter at $0.05 \mathrm{AU}$. However, their calculated atmospheric pressures are not much different from Yelle's (2004) values, therefore, we use the results from Yelle (2004) which cover a wide range of possible exoplanet locations.

One can see that the ionospheric density increases if the hydrogen-rich gas giant is located at a closer orbital distance. This ion enrichment is related to a higher stellar EUV flux at a closer distance to a star. Exoplanets at closer orbital distances are also exposed to more extreme stellar plasma flows but, on the other hand, the upper atmospheres of planets under such extreme conditions are also much more strong ionized. By using the stellar and planetary plasma parameters shown in Table 4, we estimate the corresponding ram and planetary ion pressures which are summarized in Table 5.

As one can see from Table 5, the ion pressure of a "Hot Jupiter" can easily balance the incoming stellar wind and average expected CME ram pressures at $\sim 2.3 r_{\mathrm{pl}}$ above the visual radius, that is close to but above the critical ion pick-up erosion distance where $P_{\text {tot }}$ reaches $\approx 10^{-4} \mathrm{dyn} \mathrm{cm}^{-2}$. We note that a factor of 2 related to the estimated ionopause pressure is within the uncertainties of atmospheric/ionospheric model assumptions. From these estimates we can conclude that the ionopause of a "Hot Jupiter" when it is exposed to ordinary stellar winds or average CMEs will form at a location where such a massive exoplanet will not be strongly eroded by the stellar plasma flow. On the other hand, one can also see from Table 5 
that the ion pressure is not able to balance the ram pressure of fast CMEs between orbital distances of $\sim 0.02-0.1$ AU. Panel (c) in Fig. 6 shows that the CMEs reach their highest ram pressure at about $0.02 \mathrm{AU}$, therefore the ram pressure is lower at orbital distances $\lesssim 0.02$ AU.

Our rough estimates indicate that CMEs at distances $>0.015 \mathrm{AU}$ and $<0.045 \mathrm{AU}$ have ram pressures that are too large to be balanced by the pressure of the planetary atmosphere. In such a case the CME plasma flow will compress the planetary atmosphere until it is approximately hydrostatic. This should be in agreement with Garcia-Munoz (2007) and Murray-Clay et al. (2009) who mentioned that planetary wind (and an expanded atmosphere) will be confined if the stellar wind pressure is larger than the pressure at the planetary wind's sonic radius. Because the escape time for the wind is comparable to the CME timescale, the atmosphere may readjust during the CME passing to its hydrostatic equilibrium configuration. This new steady state will be characterized by higher atmospheric pressures and closer locations of the planetary obstacle together this may result in high non-thermal mass loss rates (Khodachenko et al. 2007). A detailed study of the efficiency of CME collisions with "Hot Jupiters" is beyond the scope of this work, but will be addressed in future studies.

Interestingly, the orbital distance where fast CMEs should efficiently erode the atmospheres of "Hot Jupiters" corresponds to the orbital distance shown in Fig. 4, where so far no exoplanets within the mass range between $0.1 M_{\text {Jup }}-1.0 M_{\text {Jup }}$ have been discovered. One may wonder if this is a coincidence, or if fast CMEs are responsible for the missing "Hot Jupiters" between 0.02-0.035 AU (see Fig. 4). In future studies, we plan to investigate if fast CMEs could be a reason for the missing exoplanet population within the abovementioned mass range and orbital distances.

\section{Conclusion}

We applied a modified energy-limited mass loss equation which can reproduce the full hydrodynamic approach of Penz et al. (2008a) for the study of the thermal mass loss from 57 known transiting exoplanets over $4 \mathrm{Gyr}$. We note also that previous thermal atmospheric mass loss evolution studies, like those of Lammer et al. (2003), Baraffe et al. (2004), Lecavelier des Etangs (2007), Hubbard et al. (2007a,b) and Davis \& Wheatley (2009), which applied energy-limited formulas but neglected that the heating efficiency is substantially less than $100 \%$, overestimated the mass loss rates. We studied the initial mass of these exoplanets and found that for the given planetary and stellar parameters, only the low density "Hot Jupiter" WASP-12b could have lost about $6-12.5 \%$ of its initial mass. There are also several transiting planets like the low density exoplanet CoRoT$1 \mathrm{~b}$ which lost about $1.3-4 \%$ of their initial masses. Due to the Roche lobe effect the mass loss from close-in gas giants can be very efficient at orbital distances $\leq 0.02 \mathrm{AU}$. However, most of the observed transiting exoplanets experienced negligible mass loss during their lifetime. Compared to high density exoplanets, low density gas giants are affected much more strong by thermal mass loss. The mass loss enhancing effect of the Roche lobe together with low planetary densities are the main factors for high mass loss rates. Depending on the heating efficiency, low density Neptune-class objects can lose their hydrogen envelopes at orbital distances $\leq 0.02 \mathrm{AU}$.

Our study indicates that one can rule out that the first discovered transiting "Super-Earth" CoRoT-7b is a remnant of a thermally evaporated hot gas giant. But one cannot rule out that
CoRoT-7b is the core of a low density Neptune-class or subNeptune-class exoplanet that lost its hydrogen envelope. Owing to the EUV flux evolution and the Roche lobe effect, the thermal mass loss is lower from exoplanets orbiting low mass stars, compared to planets at similar orbital distances around G- or Ftype stars. We found that non-thermal stellar plasma-induced $\mathrm{H}^{+}$ pick-up erosion of a non- or weakly magnetized "Hot Jupiter" is most likely negligible if such a planet interacts with the ordinary stellar wind or average CMEs. Due to the large stellar EUV flux, extended ionospheres are produced. The ion pressure in these ionospheres is strong enough so that the stellar wind and CME ram pressure can be balanced at distances of a few planetary radii, resulting in negligible atmospheric erosion rates over evolutionary timescales. Fast CMEs, however, cannot be balanced by the planetary ion pressure at orbital distances between $\sim 0.02-$ 0.1 AU. During such collisions, "Hot Jupiters" may experience high non-thermal escape rates. Future research on fast CME interaction with "Hot Jupiters" will help us to understand if such extreme events are behind the phenomenon that no gas giants with masses $<1.0 M_{\text {Jup }}$ have been discovered so far at orbital distances $\leq 0.035 \mathrm{AU}$. Finally, we conclude that the results of our study show that the discovery of transiting exoplanets at orbital distances $\leq 0.015 \mathrm{AU}$ and ground-based follow-up mass determinations together with theoretical mass loss studies can bring reliable information on the statistics the of remaining cores of shrinked gas giants.

Acknowledgements. The authors thank the anonymous referee for constructive comments and suggestions which helped to improve the paper. H. Lammer, P. Odert, M. Leitzinger, M. L. Khodachenko and A. Hanslmeier gratefully acknowledge the Austrian Fonds zur Förderung der wissenschaftlichen Forschung (FWF grant P19446) for supporting this project. M. Panchenko and M. L. Khodachenko acknowledge also the Austrian Fonds zur Förderung der wissenschaftlichen Forschung (project P20680-N16). H. Lammer, H. I. M. Lichtenegger, H. K. Biernat, Yu. N. Kulikov and N. V. Erkaev thank the AAS "Verwaltungsstelle für Auslandsbeziehungen" and the RAS. H. Lammer, H. I. M. Lichtenegger, M. L. Khodachenko and Yu. N. Kulikov acknowledge support from the Helmholtz-Gemeinschaft as this research has been supported by the Helmholtz Association through the research alliance "Planetary Evolution and Life". H. Lammer, M. L. Khodachenko, T. Penz, and Yu. N. Kulikov also acknowledge the International Space Science Institute (ISSI; Bern, Switzerland) and the ISSI teams "Evolution of Habitable Planets" and "Evolution of Exoplanet Atmospheres and their Characterization". H. K. Biernat acknowledges additional support due to the Austrian Science Fund under project P20145-N16. The authors also acknowledge fruitful discussions during various meetings related to the Europlanet N2 activities as well as within the N2 Exoplanet discipline working group DWG 7. T. Penz and G. Micela acknowledge support by the Marie Curie Fellowship Contract No. MTKD-CT-2004002769 of the project "The influence of stellar high radiation on planetary atmospheres". The authors also thank the Austrian Ministry bm:bwk and ASA for funding the CoRoT project.

\section{References}

Baraffe, I., Selsis, F., Chabrier, G., et al. 2004, A\&A, 419, L13

Burrows, A., Guillot, T., Hubbard, W. B., et al. 2000, ApJ, 534, L97

Cecchi-Pestellini, C., Ciaravella, A., \& Micela, G. 2006, A\&A, 458, L13

Cecchi-Pestellini, C., Ciaravella, A., Micela, G., et al. 2009, A\&A, 496, 863

Chassefíere, E. 1996a, J. Geophys. Res., 101, 26039

Chassefíre, E. 1996b, Icarus, 124, 537

Davis, T. A., \& Wheatley, P. J. 2009, MNRAS, 396, 1012

Erkaev, N. V., Penz, T., Lammer, H., et al. 2005, ApJS, 157, 396

Erkaev, N. V., Kulikov, Yu., Lammer, H., et al. 2007, A\&A, 472, 329

Evans, J. V. 1977, Rev. Geophys., 15, 325

Grießmeier, J.-M., Stadelmann, A., Penz, T., et al. 2004, A\&A, 425, 753

Guhathakurta, M., Holzer, T. E., \& MacQueen, R. M. 1996, ApJ, 458, 817

Guillot, T. 2005, Ann. Rev. Earth Planet. Sci., 33, 493

Hubbard, W. B., Hattori, M. F., Burrows, A., et al. 2007a, ApJ, 658, L59

Hubbard, W. B., Hattori, M. F., Burrows, A., et al. 2007b, Icarus, 187, 358

Kasting, J. F., \& Pollack, J. B. 1983, Icarus, 53, 479

Khodachenko, M. L., Lammer, H., Lichtenegger, H. I. M., et al. 2007, Planet Space Sci., 55, 631 
Kliore, A. J., \& Luhmann, J. G. 1997, J. Geophys. Res., 96, 21, 281

Koskinen, T. T., Aylward, A. D., \& Miller, S. 2007, Nature, 450, 845

Lammer, H., Selsis, F., Ribas, I., et al. 2003, ApJ, L121, 598

Lammer, H., Lichtenegger, H. I. M., Biernat, H. K., et al. 2006, Planet. Space Sci., 54, 1445

Lara, A., González-Esparza, J. A., \& Gopalswamy, N. 2004, Geofísica Internacional, 43, 75

Lecavelier des Etangs, A. 2007 A\&A, 461, 1185

Lecavelier des Etangs, A., Vidal-Madjar, A., McConnell, J. C., et al. 2004, A\&A, 418, L1

Léger, A., Rouan, D., Schneider, J., et al. 2009, A\&A, 506, 287

Maloney, P. R., Hollenbach, D. J., \& Tielens, A. G. G. M. 1996, ApJ, 466, 561

Muñoz García, A. 2007, Planet. Space Sci., 55, 1426

Murray-Clay, R. A., Chiang, E. I., \& Murray, N. 2009, ApJ, 693, 23

Penz, T., \& Micela, G. 2008, A\&A, 479, 579

Penz, T., Erkaev, N. V., Kulikov, Yu. N., et al. 2008a, Planet. Space Sci., 56, 1260

Penz, T., Micela, G., \& Lammer, H. 2008b, A\&A, 477, 309
Phillips, J. L., \& McComas, D. J. 1991, Space Sci. Rev., 55, 1 Preibisch, T., \& Feigelson, E. D., 2005, ApJS, 160, 390

Ribas, I., Guinan, E. F., Güdel, M., et al. 2005, ApJ, 622, 680

Sheeley, N. R., Jr., Wang, Y.-M., Hawley, S. H., et al. 1997, ApJ, 484, 472

Sekiya, M., Nakazawa, K., \& Hayashi, C. 1980a, Earth Planet. Sci. Lett., 50, 197

Sekiya, M., Nakazawa, K., \& Hayashi, C. 1980b, Prog. Theoret. Phys., 64, 1968

Sekiya, M., Hayashi, C., \& Nakazawa, K. 1981, Prog. Theoret. Phys., 66, 1301

Tian, F., Toon, O. B., Pavlov, A. A., et al. 2005, ApJ, 621, 1049

Vidal-Madjar, A., Lecavelier des Etangs, A., Desert, J.-M., et al. 2003, Nature, 422,143

Waite Jr., J. H., Cravens, T. E., Kozyra, J., et al. 1983, J. Geophys. Res., 88, 6143

Watson, A. J., Donahue, T. M., \& Walker, J. C. G. 1981, Icarus, 48, 150

Yelle, R. V. 2004, Icarus, 170, 167

Yelle, R. V. 2006, Icarus, 183, 508

Zhang, M. H. G., Luhmann, J. G., Kliore, A. J., et al. 1990, J. Geophys. Res. 95, 14,829

Zhang, T. L., Delva, M., Baumjohann, W., et al. 2007, Nature, 450, 654 